\title{
Dengue Hemorrhagic Fever in an Adult Traveler Returning to Japan
}

\author{
Koji Goto ${ }^{1}$, Shuji Hatakeyama ${ }^{1}$, Koh Okamoto ${ }^{1}$, Takatoshi Kitazawa ${ }^{1,3}$, Katsutoshi Abe ${ }^{1}$, \\ Kyoji Moriya ${ }^{1}$, Kazuhiko Koike ${ }^{2}$ and Hiroshi Yotsuyanagi ${ }^{1}$
}

\begin{abstract}
We report the case of a Japanese traveler who developed dengue hemorrhagic fever (DHF) with a probable secondary infection with dengue virus type 2 (DENV-2). DHF usually occurs in children, and rarely in adult travelers. Proper and timely interventions can markedly reduce the mortality rate of DHF patients. The expansion of endemic areas and increased frequency of travel to these areas may suggest increased incidence of DHF in non-endemic areas in the near future. Early recognition of reinfection with dengue virus and warning signs of circulatory failure are crucial to prevent a severe shock state.
\end{abstract}

Key words: dengue hemorrhagic fever (DHF), dengue shock syndrome (DSS), plasma leakage phase, antibody-dependent enhancement

(Intern Med 51: 1779-1782, 2012)

(DOI: 10.2169/internalmedicine.51.7397)

\section{Introduction}

Dengue virus (DENV) causes dengue fever (DF), characterized by the sudden onset of fever and nonspecific symptoms such as headache, myalgia, arthralgia, rash, and gastrointestinal symptoms, together with characteristic laboratory findings such as leukopenia, thrombocytopenia, and elevated levels of transaminase $(1,2)$. Cutaneous petechiae and mucosal hemorrhage such as epistaxis, gingival bleeding, and gastrointestinal bleeding may also occur in some cases. Dengue usually goes into spontaneous remission after approximately 1 week, whereas this disease can exhibit a wide range of clinical presentations - from asymptomatic or uncomplicated DF to severe manifestations known as dengue hemorrhagic fever (DHF) or dengue shock syndrome (DSS) (3).

DENV, which is transmitted by Aedes mosquitoes (4), is a positive-sense, single-stranded RNA virus belonging to the genus Flavivirus of the family Flaviviridae, and has four serotypes: dengue virus types $1,2,3$ and 4 (DENV-1, DENV2, DENV-3 and DENV-4) (5). The regions in which the vi- rus is endemic have extended from rural districts to urban areas, and the incidence of DF has been increasing rapidly over the past few decades owing to lifestyle changes after World War II and the development of transportation. Currently, the disease is endemic in more than 100 countries and regions, many of which are located in Southeast Asia, and approximately 50-100 million cases of DF occur worldwide each year (1).

DHF/DSS are usually seen in children of endemic areas (6) and rarely in adults, especially in adult travelers (3). However, a secondary infection, particularly with the Asiangenotype strain of DENV-2 $(7,8)$, may increase the risk of DHF/DSS even in adults (5). Here, we report a case of DHF in an adult traveler, who had repeatedly traveled to Southeast Asia, with a probable secondary DENV-2 infection. Although a revised dengue classification has recently been proposed by World Health Organization (WHO) and is now under evaluation (9), we mainly used the traditional classification (2) that allows comparison with the numerous previous reports.

\footnotetext{
${ }^{1}$ Department of Internal Medicine, Infectious Diseases, Graduate School of Medicine, The University of Tokyo, Japan, ${ }^{2}$ Department of Internal Medicine, Gastroenterology, Graduate School of Medicine, The University of Tokyo, Japan and ${ }^{3}$ Department of Internal Medicine, Teikyo University School of Medicine, Japan

Received for publication January 25, 2012; Accepted for publication March 19, 2012

Correspondence to Dr. Hiroshi Yotsuyanagi, hyotsu-tky@umin.ac.jp
} 
Table 1. Laboratory Data on Admission

\begin{tabular}{crcr}
\hline Peripheral blood & \multicolumn{3}{c}{ Blood chemistry } \\
WBC & $2.3 \times 10^{3} / \mu \mathrm{L}$ & Alb & $3.8 \mathrm{~g} / \mathrm{dL}$ \\
Neutrophil & $79.0 \%$ & AST & $66 \mathrm{IU} / \mathrm{L}$ \\
Eosinophil & $0.0 \%$ & ALT & $31 \mathrm{IU} / \mathrm{L}$ \\
Basophil & $1.0 \%$ & T-Bil & $0.6 \mathrm{mg} / \mathrm{dL}$ \\
Monocyte & $6.0 \%$ & BUN & $19.7 \mathrm{mg} / \mathrm{dL}$ \\
Lymphocyte & $14.0 \%$ & Cre & $1.03 \mathrm{mg} / \mathrm{dL}$ \\
Others & $0.0 \%$ & CRP & $1.98 \mathrm{mg} / \mathrm{dL}$ \\
RBC & $478 \times 10^{4} / \mu \mathrm{L}$ & & \\
Hb & $14.2 \mathrm{~g} / \mathrm{dL}$ & Coagulation test & \\
Ht & $42.4 \%$ & PT-INR & 1.29 \\
Plt & $8.3 \times 10^{4} / \mu \mathrm{L}$ & aPTT & $48.0 \mathrm{~s}$ \\
& & Fbg & $311 \mathrm{mg} / \mathrm{dL}$ \\
\hline
\end{tabular}

RBC, red blood cell; Ht, hematocrit; Plt, platelet; Alb, albumin; AST, aspartate aminotransferase; ALT, alanine aminotransferase; T-Bil, total bilirubin; BUN, blood urea nitrogen; Cre, creatinine; CRP, C-reactive protein; PT-INR, prothrombin time-international normalized ratio; aPTT, activated partial thromboplastin time; Fbg, fibrinogen
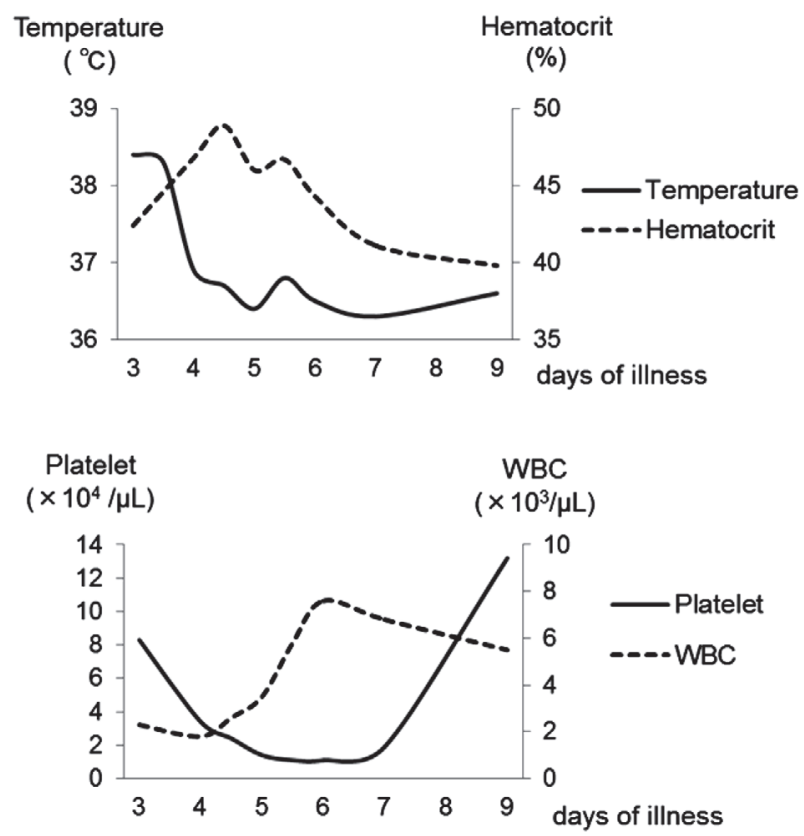

Figure 1. Clinical course of dengue illness in the present patient.

\section{Case Report}

A 51-year-old man presented with a two-day history of a fever of $39.5^{\circ} \mathrm{C}$, maculopapular rashes, diarrhea, malaise, nausea, and loss of appetite. He had stayed in Myanmar mainly in rural villages for 10 days, and the fever appeared just on returning to Japan. He had repeatedly traveled to stay in Myanmar for research, and had previously been diagnosed with DENV infection.

Blood examinations (Table 1) revealed bicytopenia (white blood cell count, $2,300 / \mu \mathrm{L}$; platelet count, $8.3 \times 10^{4} / \mu \mathrm{L}$ ), abnormal liver function (aspartate aminotransferase level, 66 IU/L; alanine aminotransferase level, 31 IU/L), coagulopathy (prothrombin time-international normalized ratio, 1.29; activated partial thromboplastin time, $48.0 \mathrm{~s}$ ), and hypoalbumi- nemia (albumin level, $3.8 \mathrm{~g} / \mathrm{dL}$ ).

DENV-2 genome was detected in the blood and urine samples by reverse-transcription polymerase chain reaction assay. Anti-DENV IgM antibodies were negative (index 0.62 ), but anti-DENV IgG antibodies were positive (index 1.98) by an enzyme-linked immunosorbent assay (ELISA) at 3 days after the onset of illness. No other pathogens such as malaria, influenza virus were detected and bacterial blood cultures were negative.

His blood pressure was $100-110 / 75-80 \mathrm{mmHg}$ with a heart rate of 90 beats per minute, but preshock-like symptoms such as momentary loss of consciousness were observed prior to hospitalization; therefore, intravenous fluid therapy (1-1.2 $\mathrm{mL} / \mathrm{kg}$ body weight/hour) was conducted on admission to the hospital. Although the patient was unable to tolerate oral fluid, intravenous fluid therapy was started at the decreased maintenance rate compared with the recommended regimen (1.5-2.0 mL/kg/hour), and closely monitored vital signs and hematocrit, because circulatory disturbance observed was not so severe (9).

The fever had continued for 4 days and subsided on the second day of hospitalization. However, thrombocytopenia rapidly proceeded to $10 \times 10^{3} / \mu \mathrm{L}$ at the minimum, and even with fluid replacement, the hematocrit increased by $23 \%$ from his baseline (Fig. 1). Pleural effusion and ascites were also observed (Fig. 2) and the patient felt light-headed on standing. Bleeding tendencies such as epistaxis appeared. These findings indicated that the patient's condition had advanced to the plasma leakage phase. Persistent fever, symptoms of plasma leakage (hemoconcentration, pleural effusion and hypoalbuminemia), severe thrombocytopenia, and bleeding tendencies (petechiae and epistaxis) led to the diagnosis of grade II DHF (2).

Intravenous fluid therapy was stopped when the recovery of appetite, decrease in hematocrit, increase in urine output, and rise in blood pressure were observed. The white blood cell count exhibited a minimum of $1,800 / \mu \mathrm{L}$ on day 4 of his illness and began to rise after the fever subsided. The platelet count began to rise on day 7 of his illness after reaching $1.0 \times 10^{4} / \mu \mathrm{L}$, which was consistent with the improvement of plasma leakage symptoms. By day 9 of his illness, the platelet count recovered to $13 \times 10^{4} / \mu \mathrm{L}$. Although the platelet count fell to $1.0 \times 10^{4} / \mu \mathrm{L}$, platelet transfusion was not given because serious bleeding symptoms (e.g., gastrointestinal bleeding) were not observed. After the transaminase level peaked on days 5-6 of his illness (aspartate aminotransferase level, 96 IU/L; alanine aminotransferase level, 41 IU/L), they remained at approximately $60 \mathrm{IU} / \mathrm{L}$ throughout the period of hospitalization. The patient was discharged without exhibiting any complication.

\section{Discussion}

DHF/DSS is reported in $1.0-3.0 \%$ of all cases of dengue $(10,11), 95 \%$ of which occur in children less than 15 years of age (6), and rarely in adult travelers (3). In Japan, 

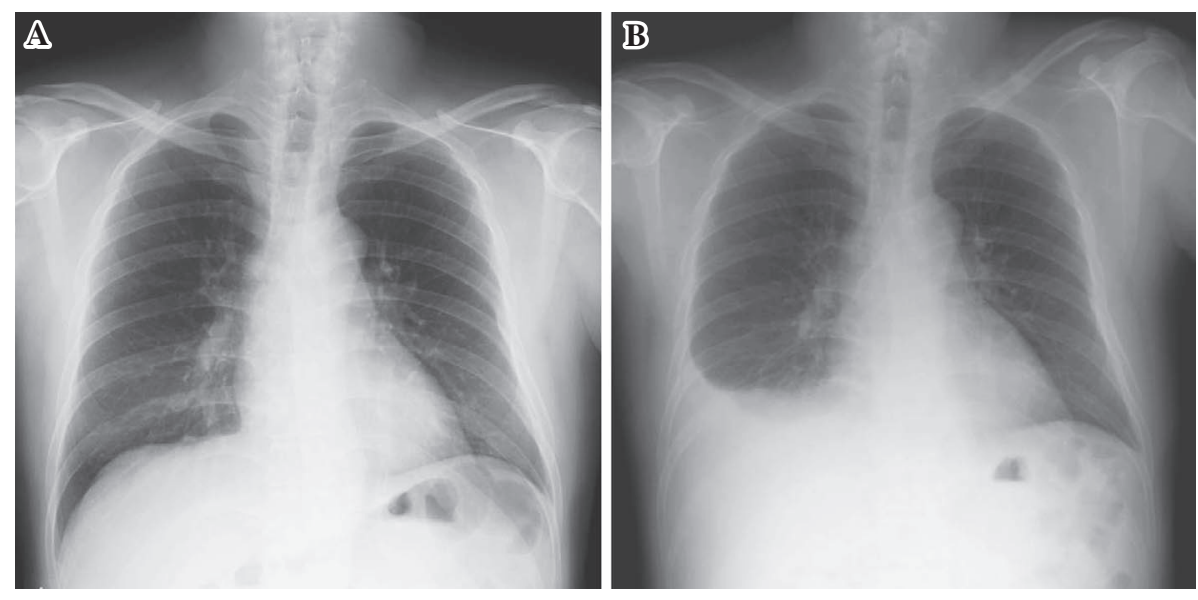

Figure 2. Chest radiographs. (A) Chest radiograph without pleural effusion on day 3 of his illness.

(B) Chest radiograph with pleural effusion on the right side on day 5 of his illness.

as few as 50 to 100 cases of DF have been reported each year, whereas 245 cases in 2010. Travel-related dengue has been only seen in Japan ever since the epidemic between 1942 and 1945 (12). A case of DHF/DSS in a Japanese traveler was first documented in 1989 (13) and five adult Japanese cases of DHF/DSS have been reported to date in the literature.

The disease course of DHF is divided into three phases: febrile, plasma leakage (critical), and convalescent (recovery) phases $(9,14)$. Loss of plasma volume owing to an increased vascular permeability is the principal pathophysiology, and adequate fluid replacement results in a favorable outcome $(2,15)$. We predicted the disease progression from the patient's history of DF, the increased hematocrit and persistent thrombocytopenia. We could prevent the development of severe circulatory failure by early and effective fluid administration based on the frequent assessment of vital signs, urine output and hematocrit.

The classical WHO guideline has classified dengue into DF and DHF/DSS (2). However, the relevance of this guideline has been debated because the main criteria such as hemoconcentration may often be accessed only retrospectively or plasma leakage can occur without thrombocytopenia or hemorrhage, which leads to misclassification of apparently severe cases as not severe (16). Thus, WHO revised the guideline in 2009, which reclassifies dengue into dengue with or without warning signs and severe dengue (9).

The current patient presented with fluid accumulation, increase in hematocrit concurrent with rapid decrease in platelet count, and mucosal bleeding whereas circulatory disturbance, fluid accumulation, or bleeding was not so severe as to be fatal. In addition, he had no severe organ involvement. These findings led to diagnosis of 'dengue with warning signs' under the new guideline, whereas delayed intervention may possibly lead to progression into severe dengue.

It has been suggested that a secondary infection with a DENV serotype different from that causing the primary infection increases the risk of DHF/DSS through antibody- dependent enhancement $(\mathrm{ADE})(17,18)$ and so-called 'original antigenic sin' $(19,20)$. Infection with one serotype confers life-long protective immunity to that same serotype, but only temporary and incomplete immunity against other serotypes. The resultant non-neutralizing antibodies as well as cross-reactive CD4+ and CD8+ $\mathrm{T}$ cells might lead to progression into DHF/DSS $(17,20)$.

The present patient had repeatedly traveled to stay in Myanmar for research, and had previously been diagnosed with DENV infection. Thus, this was considered as a probable secondary infection. Secondary infections are characterized by high hemagglutination inhibition titers or elevated IgG levels by ELISA in acute specimen (21). The $\operatorname{IgM} / \operatorname{IgG}$ antibody ratio is also a valuable diagnostic tool when the patient has become positive for IgM antibodies which can be sometimes low or absent in secondary infections (21). The present case had IgG antibodies against DENV without a detectable IgM response as early as 3 days after the onset of illness, which was additionally indicative of a DENV secondary infection. However, serological cross-reactivity across closely related flaviviruses is frequently observed. The patient had no history of infection with other flaviviruses such as Japanese encephalitis virus (JEV) or yellow fever virus (YFV), whereas he had been vaccinated against JEV during childhood (but never YFV), and a possible influence of the vaccination could not be denied.

It has also been indicated that the severity of the disease depends on the serotype of the infecting virus (1). A secondary infection with DENV-2, especially the Asian-genotype strain of DENV-2, may induce stronger cross-reactive responses than other serotypes (22), and is closely associated with DHF/DSS $(7,, 8)$. The infection of DENV-2 in Myanmar suggested that the infecting virus might be the Asiangenotype strain. There was a possibility that this might be the reason why the present patient developed DHF even though he was an adult traveler. This patient has no comorbid conditions such as diabetes mellitus and renal failure that may make dengue more complicated.

Here, we reported the case of a Japanese traveler who de- 
veloped DHF with a probable secondary infection with DENV-2 and required inpatient management. Although only a few cases of dengue have been reported to date in Japan, considering the worldwide expansion of endemic areas and the increasing number of frequent travelers to these endemic areas, future increases in the number of patients in nonendemic regions such as Japan are predicted. Most cases of dengue can be managed on an outpatient basis. However, a mortality rate of $10-20 \%$ has been reported when DHF is left untreated, which can be reduced to $0.2-1.0 \%$ with appropriate inpatient management $(1,9)$. Deterioration of symptoms usually occurs around the time of defervescence (1). Appropriate management in accordance with the precise identification of the risk factors and early recognition of developing DHF/DSS is necessary.

\section{The authors state that they have no Conflict of Interest (COI).}

\section{Acknowledgement}

We are grateful to Department of Virology I, National Institute of Infectious Diseases, for the detection of DENV genome and anti-DENV antibody.

\section{References}

1. Wilder-Smith A, Schwartz E. Dengue in travelers. N Engl J Med 353: 924-932, 2005.

2. World Health Organization. Dengue Haemorrhagic Fever: Diagnosis, Treatment, Prevention and Control. 2nd ed. World Health Organization, Geneva, 1997.

3. Gibbons RV, Vaughn DW. Dengue: an escalating problem. BMJ 324: 1563-1566, 2002.

4. Gubler DJ. The global emergence/resurgence of arboviral diseases as public health problems. Arch Med Res 33: 330-342, 2002.

5. Guzmán MG, Kourí G. Dengue: an update. Lancet Infect Dis 2 : 33-42, 2002.

6. Stephenson JR. Understanding dengue pathogenesis: implications for vaccine design. Bull World Health Organ 83: 308-314, 2005.

7. Bernardo L, Izquierdo A, Prado I, et al. Primary and secondary infections of Macaca fascicularis monkeys with Asian and American genotypes of dengue virus 2. Clin Vaccine Immunol 15: 439-
446, 2008.

8. Kochel TJ, Watts DM, Halstead SB, et al. Effect of dengue-1 antibodies on American dengue-2 viral infection and dengue haemorrhagic fever. Lancet 360: 310-312, 2002.

9. Deen J, Lum L, Martinez E, Tan LH. Clinical management and delivery of clinical services. In: Dengue: Guidelines for Diagnosis, Treatment, Prevention and Control. New ed. World Health Organization, Geneva, 2009: 25-58.

10. Jelinek T, MQhlberger N, Harms G, et al. Epidemiology and clinical features of imported dengue fever in Europe: sentinel surveillance data from TropNetEurop. Clin Infect Dis 35: 1047-1052, 2002.

11. Hills SL, Piispanen JP, Humphreys JL, Foley PN. A focal, rapidlycontrolled outbreak of dengue fever in two suburbs in Townsville, north Queensland, 2001. Commun Dis Intell 26: 596-600, 2002.

12. Hotta S. Dengue epidemics in Japan, 1942-1945. J Trop Med Hyg 56: $83,1953$.

13. Okuno Y, Harada T, Ogawa M, Okamoto Y, Maeda K. A case of dengue hemorrhagic fever in a Japanese child. Microbiol Immunol 33: 649-655, 1989.

14. Malavige GN, Fernando S, Fernando DJ, Seneviratne SL. Dengue viral infections. Postgrad Med J 80: 588-601, 2004.

15. Halstead SB. Dengue. Lancet 370: 1644-1652, 2007.

16. Bandyopadhyay S, Lum LC, Kroeger A. Classifying dengue: a review of the difficulties in using the WHO case classification for dengue haemorrhagic fever. Trop Med Int Health 11: 1238-1255, 2006.

17. Halstead SB, O'Rourke EJ. Dengue viruses and mononuclear phagocytes. I. Infection enhancement by non-neutralizing antibody. J Exp Med 146: 201-217, 1977.

18. Halstead SB. In vivo enhancement of dengue virus infection in rhesus monkeys by passively transferred antibody. J Infect Dis 140: 527-533, 1979.

19. Mongkolsapaya J, Dejnirattisai W, Xu XN, et al. Original antigenic sin and apoptosis in the pathogenesis of dengue hemorrhagic fever. Nat Med 9: 921-927, 2003.

20. Fink J, Gu F, Vasudevan SG. Role of T cells, cytokines and antibody in dengue fever and dengue haemorrhagic fever. Rev Med Virol 16: 263-275, 2006.

21. Kuno G, Gómez I, Gubler DJ. An ELISA procedure for the diagnosis of dengue infections. J Virol Methods 33: 101-113, 1991.

22. Sierra B, García G, Pérez AB, et al. Long-term memory cellular immune response to dengue virus after a natural primary infection. Int J Infect Dis 6: 125-128, 2002.

(C) 2012 The Japanese Society of Internal Medicine http://www.naika.or.jp/imindex.html 